\title{
Associating Network Flows with User and Application Information
}

\author{
Ralf Ackermann ${ }^{1}$, Utz Roedig ${ }^{1}$, Michael Zink ${ }^{1}$, Carsten Griwodz ${ }^{1}$, Ralf Steinmetz ${ }^{1,2}$ \\ 1 - Darmstadt University of Technology - Industrial Process and System Communications (KOM) \\ Merckstr. 25 - 64283 Darmstadt, Germany \\ 2 - German National Research Center for Information Technology - GMD IPSI \\ Dolivo-Str.15 - 64293 Darmstadt, Germany \\ \{Ralf.Ackermann, Utz.Roedig, Michael.Zink, Carsten.Griwodz, Ralf.Steinmetz\}@KOM.tu-darmstadt.de
}

\begin{abstract}
The concept of authenticating users e.g. by means of a login process is very well established and there is no doubt that it is absolutely necessary and helpful in a multiuser environment. Unfortunately specific information about a user originating a data stream or receiving it, is often no longer available at the traversed network nodes. This applies to the even more specific question of what application is used as well. Routers, gateways or firewalls usually have to base their classification of data on IP header inspection or have to try to extract information from the packets payload.
\end{abstract}

We present an approach that works transparently and allows to associate user and application specific information with IP data streams by only slightly modifying components of the operating system environment and infrastructure components. On top of this framework we show usage scenarios for dedicatedly placing copyright information in media content and for an enhancement of the interoperation with the security infrastructure.

\section{Keywords}

Security, Network Traffic Marking, Watermarking, Firewalls

\section{INTRODUCTION AND MOTIVATION}

According to the Internet communication model, only the header information of a specific layer should be used by the network nodes to route, filter, interpret or otherwise process data. In reality though, the strict layered concept is weakened at many points and information normally assigned to different layers is used to process packets. An example is the implementation of QoS routing functions in network nodes. Information of the application layer is necessary in the router devices, which normally should only know about the network layer, to fulfill their tasks. A dedicated information, which should not only be available in its original (application) layer is the one describing the originator or receiver of a data stream. Usually only the application layer should be aware of users, but many processes within a network also benefit from this information. Example scenarios where such a knowledge is very helpful include authentication at firewall systems, logging, admission control, billing but also the placement of copyright information.

In this paper we will describe an approach to map additional information to network streams and show its implications.

\section{REQUIREMENTS}

The access to user and/or application relevant information at traversed network nodes is helpful for a number of scenarios. There is an existing classification of information types and several approaches to obtain it.

\subsection{Availability of User Information}

Some network nodes (e.g. watermarking gateways, firewalls) have to map knowledge about user identities to the data flows, to fulfill their tasks. A network node has basically two possibilities for doing that. The node can interrupt the communication path at the application layer and force the user to identify himself (explicitly, actively concerning the user part). Alternatively, the node can try to extract the information - if (still) present - by analyzing the application layer part of the communication data (implicitly, passively concerning the user part). Both methods have drawbacks:

- The active or passive gathering of user information is not always possible.

- The passive information retrieval is costly and may result in a reduction of performance. Additionally it involves a considerable implementation effort.

To avoid these drawbacks, out of band signalling can be used. The communication partners can signal user relevant information in advance before the communication data itself is sent. For using this method, a standardized protocol would be applicable and the drawbacks mentioned above could be avoided. Other problems occur though: 
With our approach it is possible to deploy the placement of the watermarking information at dedicated points that the data traffic passes through without modifying the original servers. The approach is not targeted at implementing dedicated watermarking mechanisms (which significantly differ e.g. for packaged vs. streamed content and different media) but makes use of those and parameterizes them. The parameterization info can be gathered either explicitly (e.g. because a user logs on to the service first) or implicitly by means of the analysis of network traffic dependencies (e.g. TCP requests/replies).

The scenario can be adapted for many other use cases e.g. for tracing who brought certain data (pictures, documents) into an administrative domain. In this case the mechanism has to be deployed at the receiver instead of the server side.

\subsection{Firewall Interaction}

Firewalls [2], [3] are specialized network nodes, which perform access control at network borders. They consist of packet filters, stateful filters, proxies or a combination of all these. Based on the analysis of the specifics of data traffic (using passive or active information retrieval), a firewall system decides whether packets may be passed through.

If the marking approach is used, a firewall could benefit from the information included in the flows in the following ways:

- User information: Normally, the authentication at a firewall system is performed by application-specific proxies. By using the user information, authentication proxies would not be necessary. The firewall would be able to use a generic method (uniform for all applications), to perform authentication.

- Application information: The firewall can use this information, to determine which flows together form a session. With this information, the firewall is able to handle applications which use different flows for one logical session in a generic way.

As we have shown, firewall systems can benefit from the marking approach in many aspects. Using it it would be possible to build firewalls which have a better performance than existing systems, without compromising security.

\section{CONCLUSION AND FUTURE WORK}

We have described an approach which attaches and transmits user or application specific information to network data streams. Systems do definitely have a remarkable benefit from the availability of that additional information. We consider the approach an "enabling mechanism" that can fulfill its potential especially in interaction with other existing and emerging technologies, which can be parameterized using it. The viability of the mechanisms has been determined by means of prototype implementations for the main components and will furthermore be enhanced.

\section{REFERENCES}

[1] D. Awduche, J. Malcolm, J. Agogbua, M. O'Dell, and J. McManus. Requirements for Traffic Engineering
Over MPLS. Internet Request for Comments Nr. 2702, September 1999.

[2] D. B. Chapman. Building Internet Firewalls. O'Reilly, Cambridge, 1995.

[3] W. R. Cheswick and S. M. Bellovin. Firewalls and Internet Security. Addison Wesley, 1994.

[4] A. Chiu. Authentication Mechanisms for ONC RPC. Internet Engineering Task Force, May 1999.

[5] S. Deering and R. Hinden. Internet Protocol, Version 6 (IPv6) Specification. Internet Request for Comments Nr. 2460, December 1998.

[6] W. Hua, J. Ohlund, and B. Butterklee. Unraveling the Mysteries of Writing a Winsock 2 Layered Service Provider.

[7] S. Katzenbeisser and F. (Editors). Information hiding techniques for steganography and digital watermarking. Artech House Books, 1999.

[8] S. Kent and R. Atkinson. IP Authentication Header. Internet Request for Comments $\mathrm{Nr}$. 2402, November 1998.

[9] W. Stallings. SNMP and SNMPv2 - The Infrastructure for Network Management. IEEE Communications Magazine, 36(3):37-43, March 1998.

[10] SunSoft. STREAMS Programmers Guide. SunSoft, November 1995. 